\title{
DISCHARGE FROM THE EAR: CHRONIC SUPPURATIVE OTITIS MEDIA
}
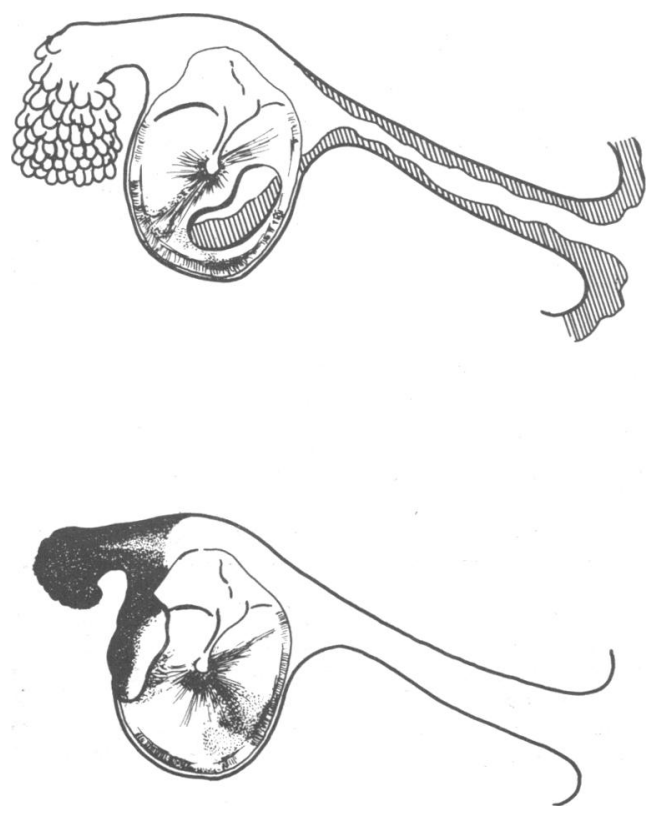

There are two types of chronic suppurative otitis media. Both present with conductive deafness and discharge without pain. In both discharge issues through a perforated drum; but one is safe and the other unsafe.

In the safe variety (tubotympanic or mucosal chronic otitis media) there is no risk of intracranial life-threatening complications. Disease affects the mucosa of the lower anterior part of the middle ear. By contrast, the unsafe variety (atticoantral) threatens meningitis, brain abscess, and other serious complications. The disease erodes the bone and cholesteatoma and chronic osteitis develop in the atticoantral region.

The perforation in the safe type is central: no matter how large, there is always a rim of drum or its annulus around the edge. In contrast, the perforation in the unsafe variety extends to the very bony edge of the drum, where chronic necrosis of the bone is often associated with the production of granulation tissue. This marginal perforation is usually posterior or in the attic.

Discharge in the safe variety comes from the inflamed and secreting mucosa of the middle ear, and is mucoid. It may be intermittent, with activity provoked by water and by blockage of the Eustachian tube. In the unsafe variety the discharge is often scanty and foul smelling and there are no periods of quiescence. This discharge usually comes from the infected debris accumulating within a cholesteatoma sac. Cholesteatoma is simply skin - stratified squamous epithelium - that has entered the middle ear cleft to form a multiloculated cyst surrounding structures in the attic and extending into the air spaces connected with the mastoid antrum. When the keratin accumulating within the cholesteatoma sac becomes infected the underlying subcutaneous tissue starts to erode bone; therein lies its danger.

\section{Recognition and treatment of safe ears}
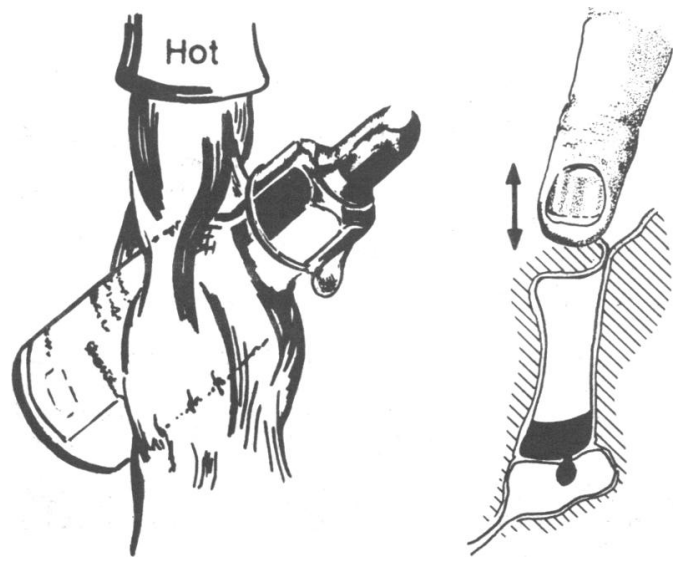

The two types of chronic suppurative otitis media can be distinguished only by carefully examining the ear drum after removing any dischargeideally with an operating microscope. More than one examination, after reducing surface inflammation with topical treatment, may be needed. Safety is suspect when the discharge smells foul, when there is granulation tissue or a polyp arising from the middle ear or outer edge of the drum, when dead skin can be aspirated from the middle ear, and when there are symptoms, such as facial weakness or vertigo, that suggest complications. Only when there is a central perforation without any of these features can safety be confirmed.

In safe ears the aim is to dry up discharge, help any hearing defect, and prevent further discharge. Drying is achieved by treating infection or allergy in the upper respiratory tract and by aural toilet to remove infected material. Rarely syringing may help: the best solution, which must be at body temperature, is half-strength Eusol in isotonic saline (mix full-strength Eusol with equal volume of $2 \times$ isotonic saline). Antibiotic-with-steroid drops are useful, and the choice of preparation may be guided by the results of swab culture. The drops must be warmed by holding the bottle under a hot tap, and massaged into the middle ear. 


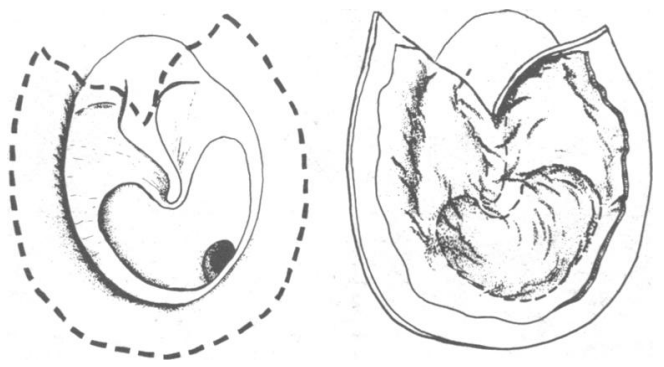

\section{Treatment of unsafe ears}
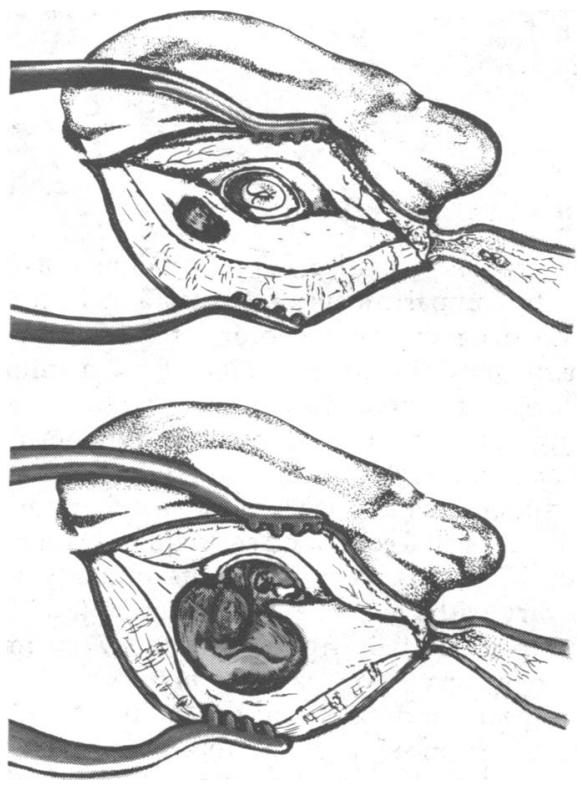

Once the ear is dry further discharge may be prevented by protecting it from water and promptly treating upper respiratory tract infection or by closing the defect in the ear drum surgically (myringoplasty). Hearing defects can be helped by a hearing aid or by reconstructing the drum and ossicular chain (tympanoplasty).

\section{Discharge from the mastoid cavity}

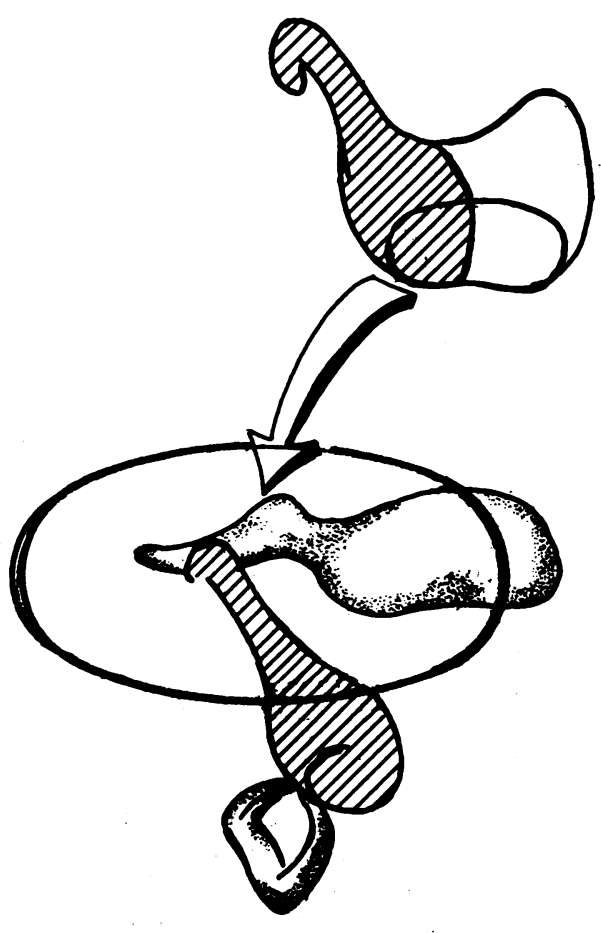

A dangerous ear must be rendered harmless before the problems due to deafness are treated. The aim of the various operations performed-radical mastoidectomy, modified radical mastoidectomy, atticotomy, atticoantrostomy-is to remove diseased and infected bone and leave a smooth wide cavity opening into a wide external ear canal. As the ear heals the cavity becomes lined with skin, which is histologically identical to cholesteatoma, but which excretes its dead squames to the exterior through wide access.

Radical mastoidectomy is an example of this kind of operation. Under general anaesthesia the ear is entered through either an endaural or postaural incision. The mastoid antrum is opened with a drill. As the opening to the antrum is enlarged it extends forward into the attic region of the middle ear. Removing the "bridge" of bone over the aditus ad antrum throws the mastoid cavity and middle ear into one. Disease is removed as the operation progresses. In classical radical mastoidectomy all the ossicular chain except the stapes is removed. The cavity is made as hemispherical as possible, without damaging the facial nerve, labyrinth, sigmoid sinus, or dura. Then the cavity is packed with, for example, ribbon gauze soaked in an antiseptic ointment. Other methods-combined approach tympanoplasty or intact canal wall techniques-avoid creating a cavity with reconstruction of the middle ear mechanism, but at the risk of enclosing residual cholesteatoma.

After operation the discharge will continue until the cavity is completely ed with skin-a process that usually takes three months and in some patients is never completed. The reason is that the discharge is exudation (since the body is wet) and the cavity lacks a waterproof lining. Continuing or recurrent discharge is due to anything that prevents or breaks down intact cavity lining. The warm damp mastoid cavity is inhospitable to healthy skin. Conditions for healing are best when operation creates as small a cavity with as wide an opening to the external meatus as possible. Even then some patients cannot form a healthy lining. Infection of discharging exudate may be a reason for this. The discharge becomes infected with Gram-negative organisms from outside or from the nasopharynx; the infected material prevents skin from healing, by its inflammatory action and by producing granulation tissue that cannot be covered by epithelium and that occludes poorly drained pockets of infected material.

During the early postoperative period the ear, protected by gauze pad or cotton wool, should be left to heal. Water must not get in. Later if there is surface infection gentle cleaning and treatment with topical antibiotics and steroids, or boric acid powder, may be successful. Much rarer causes of continuing discharge are residual disease or metaplasia of the mastoid lining to a mucus-secreting one. Treatment of deafness after an ear is safe depends on the state of the other ear. The options are to do nothing, to offer a hearing aid, or to perform reconstructive surgery (tympanoplasty). 